\title{
Accurate Estimation of Battery SOH and RUL Based on a Progressive LSTM with a Time Compensated Entropy Index
}

\author{
Taejun Bak ${ }^{1}$ and Sukhan Lee ${ }^{2}$ \\ ${ }^{1,2}$ Sungkyunkwan University, Suwon, Gyeonggi-do, 16419, Republic of Korea \\ ptjvv@skku.edu \\ lsh1@skku.edu
}

\begin{abstract}
The accurate estimation of the State of Health $(\mathrm{SOH})$ and Remaining Useful Life (RUL) has been a subject of keen interest due to its impact on safety and condition-based maintenance services. A number of approaches have been proposed to tackle this problem based either on a modeldriven or on a data-driven framework. Due to the electrochemical complexity involved in battery aging, they are yet to achieve the accuracy required, especially, for real-world applications. This is because of the difficulty either in identifying the time-varying nature of model parameters and in collecting the real-world training dataset from widely varying modes of battery usage.

In this paper, we propose a method of estimating $\mathrm{SOH}$ and RUL simultaneously in such a way as to contribute to its realworld applicability. First, noticing that battery aging causes the time sequence of charging and discharging voltage and current in a cycle to be shortened and dispersed, we define an aging index, referred to here as the time compensated entropy, for SOH and RUL. Second, for LSTM-based RUL prediction, we optimize the number of $\mathrm{SOH}$ input and the RUL prediction sequences for the minimum prediction error associated with a sequence of cycles. Third, we adopt a progressive framework of LSTMs such that whatever learned from the prior predictions are transferred to the subsequent prediction, starting with learned SOH. For experimental verification, we train the proposed progressive LSTM network based on CALCE datasets and apply to various cases of charging and discharging cycles. With $\mathrm{SOH}$ estimated online, we achieve less than 10 cycles of accuracy in RUL prediction, moving closer to real-world applicability.
\end{abstract}

\footnotetext{
Taejun Bak et al. This is an open-access article distributed under the terms of the Creative Commons Attribution 3.0 United States License, which permits unrestricted use, distribution, and reproduction in any medium, provided the original author and source are credited.
}

\section{INTRODUCTION}

The advent of lithium-ion batteries has changed our lives. The small, wireless gadgets like the smartphones and wireless devices could have been developed with the advance of lithium-ion battery technology. This technology provides us a breakthrough to overcome spatial and temporal limits and is the key to advance in Electric Vehicles (EV), Robotics and Aerospace industries.

As the importance of batteries grows, the battery management system (BMS) technology becomes more significant. The biggest disadvantage of batteries is the aging. A primary cause of battery aging is a change in the electrode and electrolyte in batteries. Solid Electrolyte Interphase (SEI) is formed on the electrode surface due to chemical reactions during the operation of batteries (Arorat, White, Carolina, \& Doyle, 1998). The SEI is becoming more unstable and increasing in quantity as the battery charges and discharges repeatedly. The growth of SEI results in capacity and power fade in batteries (Vetter, Nov, Wagner, \& Veit, 2005). Because of the aging effect which induces degradation of batteries and devices, accurate prediction or estimation on the degree of aging in batteries is necessary.

In order to manage the battery efficiently, it is necessary to accurately estimate and manage the battery State of Health (SOH) and Remaining Useful life (RUL). SOH represents the maximum discharge capacity to the rated capacity of batteries (Murnane \& Ghazel, 2017). Since the maximum discharge capacity is characteristic of battery aging, $\mathrm{SOH}$ is used as an indicator of degree of ageing. SOH of new battery with no degradation is processed in is $100 \%$. When $\mathrm{SOH}$ value reaches $80 \%$, that is the time for the replacement and can be regarded as reaching at an End of Life (EOL). RUL represents remaining life until the EOL.

It is major research subject in battery health management to estimate degree of aging in battery. There are many approaches to achieve that goal. These methods could be categorized into three methods, i.e. direct approach, modelbased approach and data-driven approach. 
In the direct approach, there are few methods using measured values directly. The coulomb counting method is a method of estimating SOH through the calculated discharge capacity by integrating the discharge current $(\mathrm{Ng}, \mathrm{Moo}$, Chen, \& Hsieh, 2009). The open circuit voltage method is estimating $\mathrm{SOH}$ using the relationship between $\mathrm{SOH}$ and OCV obtained by prior experiment. Weng, Sun, and Peng (2014) used OCV model to monitor SOC and SOH. The direct approach is easy to implement but having disadvantages of on-line estimation.

The model-based approach utilizes filtering such as kalman filters or particle filters. Liu, Yin, Song, and Peng (2018) estimate SOH using an Unscented Kalman Filter (UKF) with the health indicator as the operating time corresponding to the discharge voltage range. The filter methods have been used to predict RUL. SVR-UPF, which combines a Support Vector Regression (SVR) and an Unscented Particle Filter (UPF), has been proposed by (Peng, Zhang, Yu, \& Zhou, 2016). The model-based approach has the advantage of real time estimation, but high computational capacity is needed, and multiple tasks can't be performed on one model.

The data-driven approach uses learning models with preperformed battery measurement data. Several $\mathrm{SOH}$ estimation and RUL prediction studies based on NN structure have been conducted due to its excellent ability to learn features from the data. Especially Long Short-Term Memory (LSTM) is widely used in aging estimation model. You, Park, and $\mathrm{Oh}$ (2017) studied various methods of estimating $\mathrm{SOH}$ with LSTM structure. Zhang, Xiong, He, and Pecht (2018) proposed the model with LSTM and Monte Carlo simulation for RUL prediction and its uncertainty. This approach is based on the data collected by simulation in laboratory, i.e. voltage, current and temperature etc. It can lead to inaccurate results in real environment applications because these parameters vary depending on the battery operating environment.

We proposed a time compensated entropy index from battery voltage distribution which has low variation and a Progressive LSTM networks for SOH estimation and RUL prediction. The advantage of our approach is that it is capable of estimating $\mathrm{SOH}$ as well as predicting RUL. Many studies considered these two separately. A number of studies to predict the timing of battery replacement have been carried out under the assumption that $\mathrm{SOH}$ is known as label data. However, in a real application, BMS needs to estimate $\mathrm{SOH}$ and predicting RUL would be made through accurate $\mathrm{SOH}$ estimation. In order to perform different tasks with one model, we apply the progressive neural networks (Rusu, Rabinowitz, Desjardins, Soyer, Kirkpatrick, Kavukcuoglu, Pascanu, \& Hadsell, 2016) and combine them with LSTM for extracting features from time-series data. Knowledge for $\mathrm{SOH}$ estimation accumulated in a network is transferred to another network for predicting sequence of $\mathrm{SOH}$ accurately.

In this paper, we show a new health indicator based on information entropy for estimating $\mathrm{SOH}$. Using battery datasets from CALCE in the Maryland university, we demonstrate our progressive networks which use these indices having ability to infer the degree of aging.

Section 2 covers brief introduction to progressive neural networks. Section 3 describes our method including the time compensated entropy index and the progressive LSTM networks. In section 4, we do experiments for evaluating our methods are good at accomplishing our goal. Section 5 comes up with a conclusion.

\section{BACKGROUNDS}

Progressive neural networks are structures that have been proposed to mimic humans performing new tasks based on past experiences. Existing learning transfer models, such as transfer learning, have the disadvantage that it is impossible to transfer such knowledge that they can do various tasks. To solve these shortcomings, they use the lateral connection that leads to the existing learned network to learn the network to perform the new task (Rusu et al., 2016). It transfers the prior knowledge existing in the existing network to other networks via this connection. Through such a structure, it is possible to extract useful features for a new task.

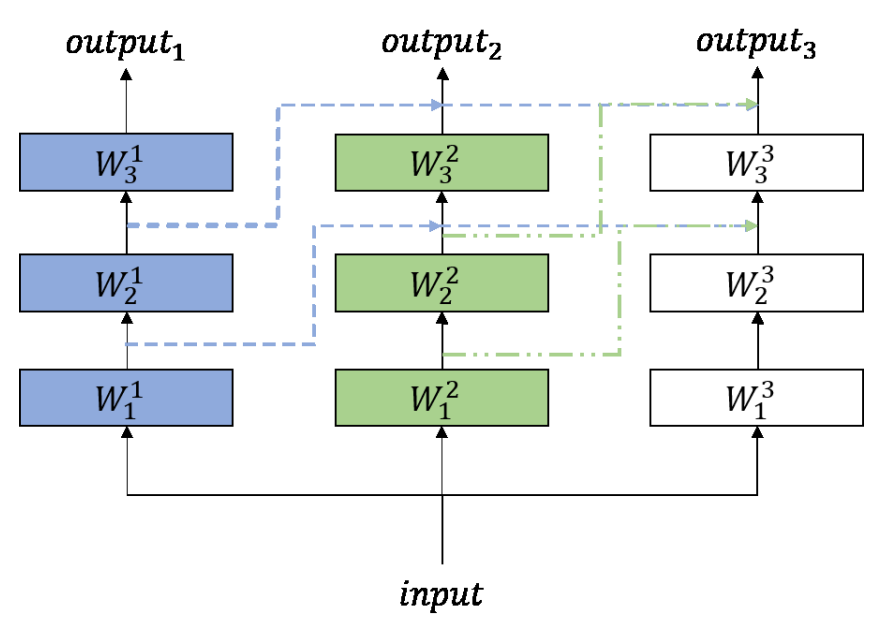

Figure 1. Progressive Neural Networks. Dashed lines are lateral connections for transferring knowledge. Each column learns to deal different tasks.

The figure 1 shows the progressive neural networks structure. The ability to integrate prior knowledge through the structure where the output is transferred to other networks on each floor of each network, hence, reduce oblivion of existing knowledge. The lateral connection in P-NN makes the network capable of doing various tasks. The ability to transfer knowledge and deal with multi-task is the reason why we use $\mathrm{P}-\mathrm{NN}$ in this paper. From the second column network in P$\mathrm{NN}$, the output of each hidden layer is written as follows: 


$$
h_{i}^{k}=f\left(W_{i}^{k} h_{i-1}^{k}+\sum_{k<j} U_{i}^{k: j} h_{i-1}^{j}\right)
$$

where $h_{i}^{k}$ is the hidden output of layer $i$ in a column $k, W_{i}^{k}$ is the weight matrix of layer $i$ in column $k$ and $U_{i}^{k: j}$ is weight matrix of lateral connection from a column $k$ to a column $j$. $f(x)$ is an activation function such as ReLU function. The learning process in P-NN is sequential. When learning the column $j$, we freeze weights of the columns before column $j$ and update weights of the column $j$ and weights of all lateral connection from column $k$ to $j$ in all $k$ less than $j$.

The progressive neural network has been applied in various fields, as it has the advantage of reusing previous results and learning new things. The performance of progressive neural networks is superior to the Transfer Learning or Deep Neural Networks as evident from the experiments on emotion recognition on learned knowledge in order to distinguish speakers and gender in (Gideon, Khorram, Aldeneh, Dimitriadis, \& Provost, 2017).

\section{Proposed Method}

Aging phenomenon of batteries is caused by physicalchemical changes inside the battery and makes the internal resistance of the battery and the maximum charge capacity be changed (Williard, He, Osterman, \& Pecht, 2013). Old batteries are characterized by a wider discharging voltage distribution than that non-aging battery's. It means changes in the distribution can be used as an indirect indicator of battery aging (Lorenzo \& Labed, 2005). In this section, the time compensated entropy index based on the battery voltage distribution and the progressive LSTM networks to infer degree of aging is proposed.

\subsection{Time Compensated Entropy Index}

Entropy in information theory is the concept that Shannon (1948) proposed to represent the amount of information that data has. Information entropy means the number of bits required to represent information. The amount of information increases as the information is chaotic and irregular.

$$
H=-\sum_{i=1}^{N} p_{i} \log _{\mathrm{b}} p_{i}
$$

Eq. (2), is a basic equation for obtaining information entropy. $p_{i}$ is the probability that an event $i$ occurs. Information entropy can be used to indicate a disorder, an uncertainty of the system. As the battery ages, the system is disordered due to the transition in the internal characteristics. Information entropy is used to represent the changes emerged as voltage distribution. A voltage in an operating cycle is represented by a histogram. Eq. (2) can be represented as Eq. (3), with the histogram which has $M$ bins.

$$
H_{k}=-\sum_{i=1}^{M} p\left(x_{i}^{k}\right) \log _{10} p\left(x_{i}^{k}\right)
$$

where $H_{k}$ is information entropy in the $k$ th battery cycle and $p\left(x_{i}^{k}\right)$ is the probability for the bin $x_{i}^{k}$ in a histogram of voltage distribution at $k$ th battery cycle. The operating time of the battery varies depending on the operating environment and variation of the information entropy may occur. Information entropy tends to decrease as operating duration is shortened. To minimize this effect, the entropy is normalized by multiplying the normalization term including the duration.

$$
\begin{gathered}
E_{k}=-\frac{\alpha}{D_{k}} \sum_{i=1}^{M} p\left(x_{i}^{k}\right) \log _{10} p\left(x_{i}^{k}\right) \\
E_{k}=-\frac{\alpha}{C_{k} \cdot D_{k}} \sum_{i=1}^{M} p\left(x_{i}^{k}\right) \log _{10} p\left(x_{i}^{k}\right)
\end{gathered}
$$

where $E_{k}$ is the time compensated entropy index. $D_{k}$ is the discharging (or charging) duration of the $k$ th cycle and we call this term a time compensated term. $\alpha$ is a length coefficient and can be defined as an operating time of the shortest cycle in the data. In Eq. (5), we can compensate entropy index in Eq. (4) by the discharging current additional term $C_{k}$ under the condition that discharging current changed. Discharging Time Compensated Entropy Indices and Charging Time Compensated Entropy Indices come from the voltage distribution during discharging and charging.

\subsection{Progressive LSTM model}

A progressive LSTM model (P-LSTM) with input vectors including time compensated entropy index was designed to predict battery SOH and RUL. The goal of our model is multiple performance capability to accurately estimate the $\mathrm{SOH}$ of the battery in time, predict the future $\mathrm{SOH}$ sequence through the predicted $\mathrm{SOH}$ and obtain it to the RUL. The progressive neural networks (P-NN) is a suitable structure for these objectives. The structure can effectively communicate knowledge between networks to perform multiple tasks. We have designed a P-LSTM based on LSTM that is suitable for extracting features of time series data according to the battery operation.

Figure 2 describes our models. Our model includes two PLSTM networks: 1) A network that estimates $\mathrm{SOH}$ at the current time $t$ and predict $\mathrm{SOH}$ sequences based on predicted $\mathrm{SOH}$ sequences. 2) A network that predicts future $\mathrm{SOH}$ sequences until life of batteries reaches EOL through the predicted $\mathrm{SOH}$ sequence at a first network. Both networks are based on P-LSTM architecture. The hidden state in the column is combined with the hidden state of the previous columns through the lateral connection. In P-LSTM, $h_{i}^{k}$ is the hidden state of the layer $i$ in the $k$ th column: 


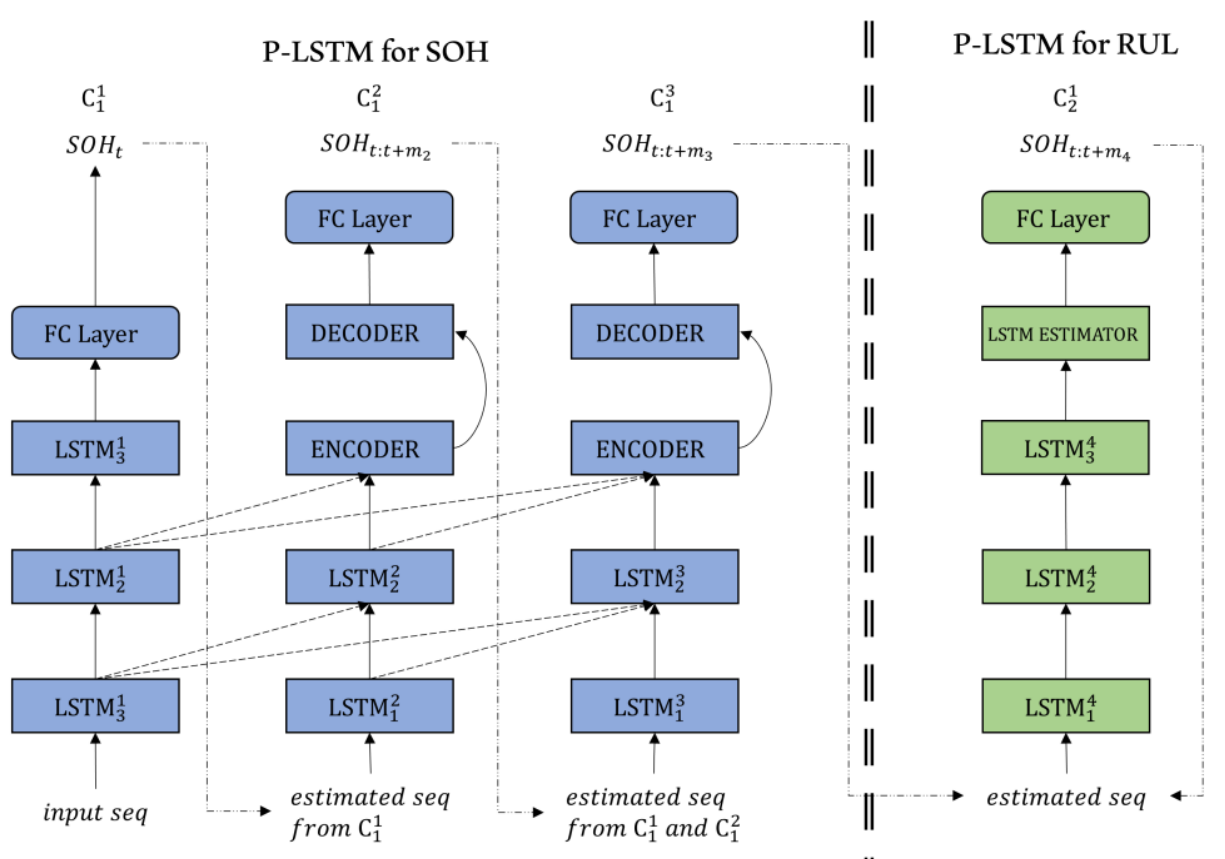

Figure 2. P-LSTM for SOH and RUL

$$
h_{i}^{k}=f\left(L_{i}^{k}\left(h_{i-1}^{k}\right)+\sum_{k<j} U_{i}^{k: j} h_{i-1}^{j}\right)
$$

where $U$ is weighted matrix in lateral connection from column $k$ to $j$ and $L_{i}^{k}$ is LSTM layer $i$ in the $k$ th column. we use ReLU function as $f(x)$.

\subsubsection{P-LSTM for SOH}

The first P-LSTM network consists of a P-NN structure with three network columns performing different roles.

The first column $\left(\mathbf{C}_{\mathbf{1}}^{\mathbf{1}}\right)$ estimates $\mathrm{SOH}$ at the current point in time. Inputs of $\mathrm{C}_{1}^{1}$ is the vector $u_{t}$ including the time compensated entropy indices. When voltage data at the cycle $t$ is given, $E_{t}=\left[\left(D E_{t}, C E_{t}\right)\right]$ is the time compensated entropy indices. where $D E_{t}$ is the discharging time compensated entropy index at the cycle $t$ and $C E_{t}$ is the charging time compensated entropy index at the cycle $t$. To specify battery's operation, input vector includes a discharge voltage distribution. For a given data set until the cycle $t$, input vector $u_{t}^{1}$ with sequence length $k_{1}$ is written as:

$$
u_{t}^{1}=\left[\left(E_{t-k_{1}-1}, P_{t-k_{1}-1}\right), \cdots,\left(E_{t}, P_{t}\right)\right]^{T}
$$

where $P_{t} \in \mathbb{R}^{n}$ is a discharge voltage distribution with $P_{t}=$ $\left(p\left(x_{1}\right), p\left(x_{2}\right), \cdots, p\left(x_{n}\right)\right)^{T}$. The input vector passes through the LSTM layer on each floor and is changed to knowledge for the next columns. After three LSTM layers and a fully connected layer, $\mathrm{SOH}$ at the cycle t $S_{t}$ as a final output is obtained. Accumulated results used as the input vector of $\mathrm{C}_{1}^{2}$.
The second column $\left(\mathbf{C}_{1}^{2}\right)$ predicts a $\mathrm{SOH}$ sequence as much as the prediction cycle $m_{2}$ from the current cycle. $C_{1}^{2}$ consists of two LSTM layers and one LSTM Enc-Dec. The Enc-Dec is responsible for predicting the $\mathrm{SOH}$ sequence of variable lengths. An input vector of $\mathrm{C}_{1}^{2}$ made from $\mathrm{C}_{1}^{1}$ is estimated SOH sequence. $u_{t}^{2}$ is input vector of the column 2 at the cycle $t$ and can be written as:

$$
u_{t}^{2}=\left[S_{t-k_{2}-1}, \cdots, S_{t}\right]^{T}
$$

where $k_{2}$ is an input sequence length of $\mathrm{C}_{1}^{2}$. The output $h_{2}^{l}$ of the LSTM layer $l$ in the second column is added to the output of the LSTM layer $l$ in $\mathrm{C}_{1}^{1}$ multiplied with the lateral connection weight. This result is used as an input to the next layer via ReLU function. Through this process, knowledge of $\mathrm{C}_{1}^{1}$, which estimates $S_{t}$ from the battery measurement data including the compensated index, is used to predict the future $\mathrm{SOH}$ sequence in $\mathrm{C}_{1}^{2}$. The output $o_{t}^{2}$ is:

$$
o_{t}^{2}=\left[\bar{S}_{t}, \cdots, \bar{S}_{t+m_{2}-1}\right]^{T}
$$

where $\bar{S}_{t}$ is predicted SOH at cycle $t$ and $m_{2}$ is a length of prediction in $\mathrm{C}_{1}^{2}$.

The third column $\left(\mathbf{C}_{1}^{3}\right)$ predicts a $\mathrm{SOH}$ sequence with longer time-length than an output of $\mathrm{C}_{1}^{2}$. Estimated $\mathrm{SOH}$ sequence from $\mathrm{C}_{1}^{1}$ and $o_{t}^{2}$ are concatenated and given as input vector $u_{t}^{3}$ as follows:

$$
u_{t}^{3}=\left[S_{t-k_{3}-m_{2}}, \cdots, S_{t-1}, \bar{S}_{t}, \cdots, \bar{S}_{t+m_{2}-1}\right]^{T}
$$


where $k_{3}$ is an input sequence length of $C_{1}^{3}$. Structure of $C_{1}^{3}$ have same structure with $C_{1}^{2}$. Outputs from the layer $l$ in $C_{1}^{1}$ and $\mathrm{C}_{1}^{2}$ are transferred to $\mathrm{C}_{1}^{3}$ via the lateral connection. $\mathrm{A} \mathrm{SOH}$ sequence with longer time can be predicted based on the knowledge to estimate $S_{t}$ from $\mathrm{C}_{1}^{1}$ and to predict future $\mathrm{SOH}$ sequences from $\mathrm{C}_{1}^{2}$. Predicted $\mathrm{SOH}$ sequence with $m_{3}$ as length of prediction in $\mathrm{C}_{1}^{3}$ can be written as:

$$
o_{t}^{3}=\left[\overline{\bar{S}}_{t}, \cdots, \overline{\bar{S}}_{t+m_{3}-1}\right]^{T}
$$

where $\overline{\bar{S}}$ is predicted $\mathrm{SOH}$ in $\mathrm{C}_{1}^{3}$.

\subsubsection{P-LSTM for RUL}

The second P-LSTM network's goal is to predict the SOH sequence from a certain point in time, which we call the starting point, to the EOL. The second P-LSTM network consists of a network column $\mathrm{C}_{2}^{1}$ with LSTM layers and LSTM estimator. $\mathrm{C}_{2}^{1}$ predicts a $\mathrm{SOH}$ sequence whose length is $m_{4}$ from input vector with $k_{4}$ of length repeatedly. $\mathrm{C}_{2}^{1}$ has the input vector $v_{t}^{1}$ which concatenates $o_{t}^{3}$ and estimated $\mathrm{SOH}$ values from $\mathrm{C}_{1}^{1}$. After predicted $\mathrm{SOH}$ sequences are larger than $k_{4}, v_{t}^{1}$ comes from results of $\mathrm{C}_{2}^{1}$ only.

For example, EOL is 510 cycle when the starting point is 300th cycle, $m_{4}$ is 40 and $k_{4}$ is 100 , the predicting process in $\mathrm{C}_{2}^{1}$ from starting point is written as:

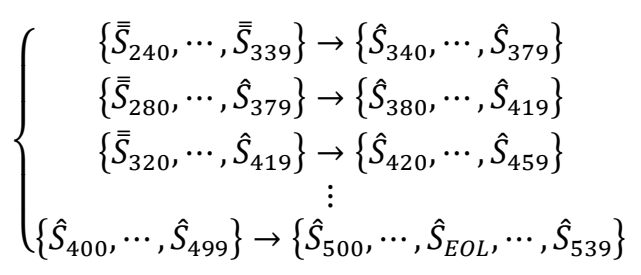

where $\hat{S}_{t}$ is predicted $\mathrm{SOH}$ from $\mathrm{C}_{2}^{1}$. Some experiments are conducted to determine model parameters like sequence lengths and output lengths.

\section{EXPERIMENTS AND RESULTS}

\subsection{Datasets}

Experiments to evaluate the performance of proposed model used data from the battery aging experiments provided by the CALCE Battery Research group at the University of Maryland (He, Williard, Osterman, \& Pecht, 2011). The dataset was created by repeatedly charging and discharging the battery over several months. The battery named CS2 used in the experiment is a lithium-ion battery with $1.1 \mathrm{Ah}$ of rated capacity. We use 6 experimental datasets conducted with CS2, i.e. CS2_33, CS2_34, CS2_35, CS2_36, CS2_37 and CS2 38. These cases are divided into two types based on discharge current. CS2_33 and CS2_34 cycled at constant discharge current of $0 . \overline{5} \mathrm{C}$ while others cycled at constant discharge current of 1C. Figure 3 shows the degeneration curves of test cases.

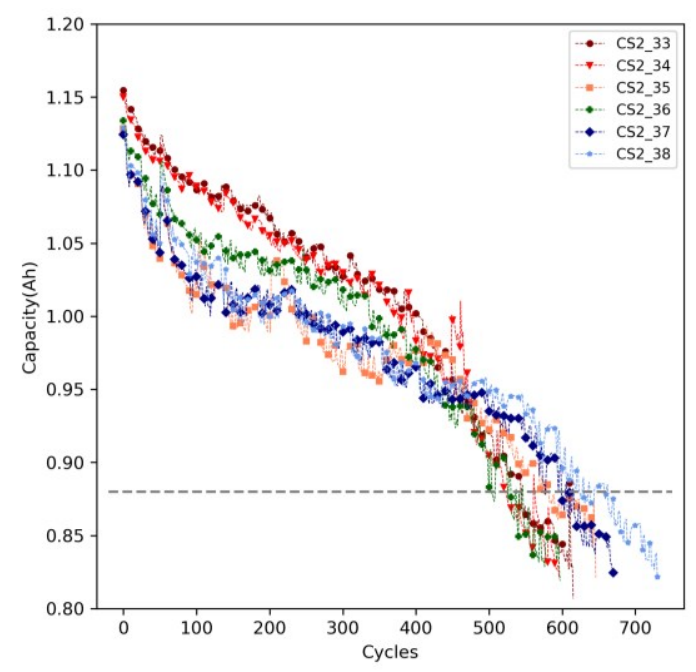

Figure 3. Degeneration curves to the vicinity of EOL from CS2 dataset. EOL is the point where curve reaches at $80 \%$ of rated capacity (dashed horizontal line).

The maximum capacity of the battery is lower as the battery operates repeatedly. EOL is where the degeneration curve reaches at a grey dashed line of $80 \%$ of rated capacity in figure 3 . The raw degeneration curve is a signal with frequent change in short period. However, this variation can interfere with estimation of SOH and RUL for test data. Therefore, a gaussian filter defined in 1-D is applied to raw degeneration curves. Filtered signals are used as label data in training data for RUL prediction.

\subsection{Model Configuration}

We conducted experiments to evaluate our method in terms of efficient estimation and prediction of the battery $\mathrm{SOH}$. The training dataset includes CS2_33, CS2_34, CS2_35, CS2_37. CS2_38 and CS2_36 are used as the validation dataset and the testing dataset, respectively. Training procedure is as follows:

1. Design the P-LSTM model with the specified parameters. The input vector is composed of the calculated time compensated entropy index, current and voltage distribution. At this time, the bin number of the voltage distribution is smaller than the size used in calculating the entropy index in order to prevent dimension of the input vector from becoming too large.

2. Train P-LSTM for SOH. First $\mathrm{C}_{1}^{1}$, which estimates $\mathrm{SOH}$ as an input vector, is learned. Second, $\mathrm{C}_{1}^{2}$ is trained by inputting the result of $\mathrm{C}_{1}^{1}$. The weight of $\mathrm{C}_{1}^{1}$ freezes while learning training $\mathrm{C}_{1}^{2}$. At last train $\mathrm{C}_{1}^{3}$ in freeze state of weights of $\mathrm{C}_{1}^{1}$ and $\mathrm{C}_{1}^{2}$.

3. Train P-LSTM for RUL. Capacity data which is label data for training P-LSTM for SOH used as training data in this step. 


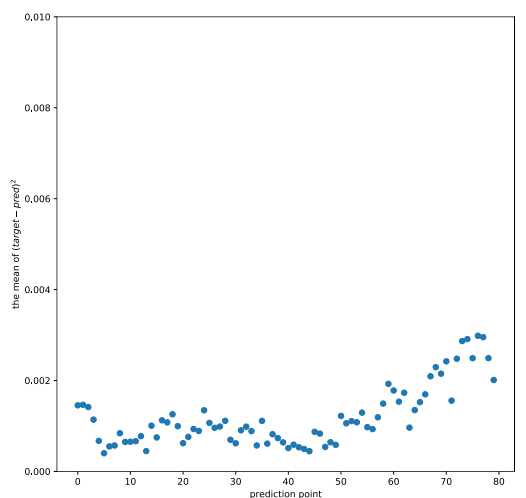

(a) sequence length: 80

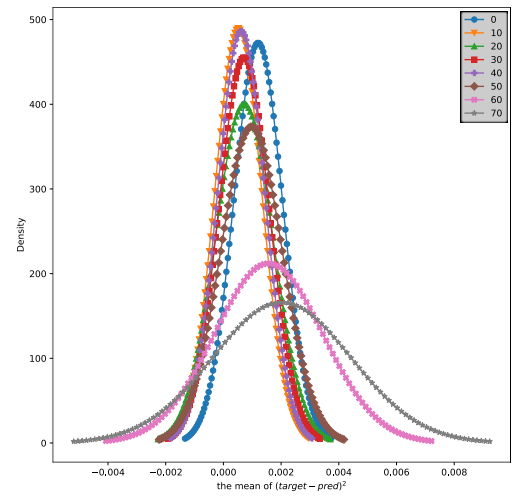

(d) sequence length: 80

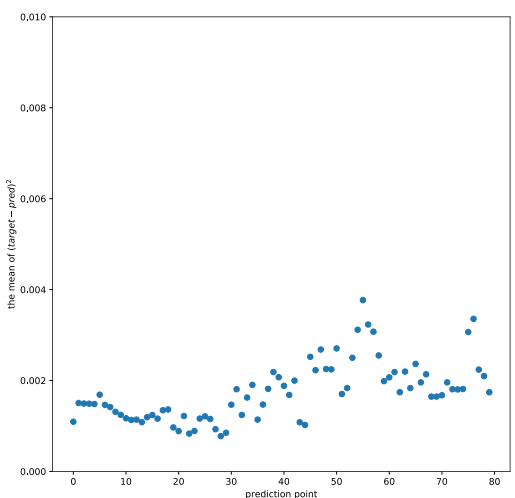

(b) sequence length: 100

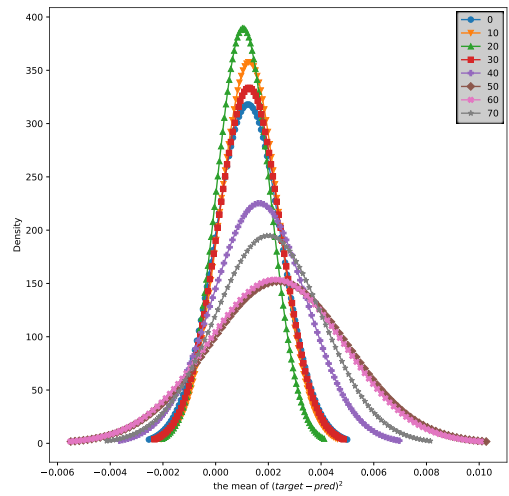

(e) sequence length: 100

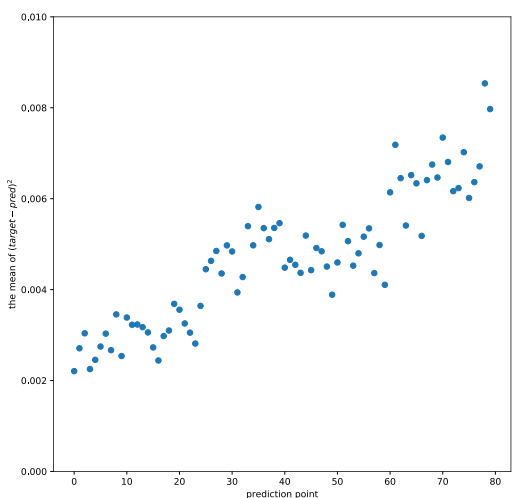

(c) sequence length: 120

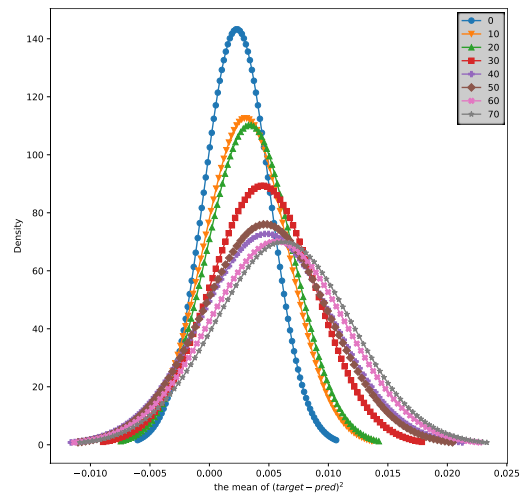

(f) sequence length: 120

Figure 4. The average of errors on each prediction points are described in the first row. The distributions of error (d), (e) and (f) widen according to increasing of prediction length. Optimal sequence length is 80 , which shows good performance in terms of error and variance.

The optimizer for model learning uses AdamOptimizer (Kingma \& $\mathrm{Ba}, 2015$ ). The parameters for the P-LSTM configuration is listed in Table 1. Parameters selection, such as sequence lengths, determines the performance of the model. But for most applications the parameters are determined by empirical methods. To determine parameters in P-LSTM, we conduct a simple experiment through one column used in P-LSTM to efficiently determine the seq length and prediction cycle. The average and variance of the error according to the input sequence length and prediction length are applied to choose parameters.

The experiment was performed on the same model as $\mathrm{C}_{2}^{1}$ with size of hidden states is 32 . Three experiments were performed in which the prediction cycle was fixed at 80 cycles and the sequence length was changed to 80,100 and 120 . The networks learn from training datasets and evaluate the accuracy with prediction at several cycles from 100 to 400 at 50 intervals with the test datasets. The results are listed in the Table 2.
Table 1. Parameters.

\begin{tabular}{|c|c|}
\hline Parameter & Value \\
\hline$k_{1}, k_{2}$ & 20 \\
\hline$k_{3}$ & 40 \\
\hline$k_{4}$ & 80 \\
\hline$m_{2}$ & 20 \\
\hline$m_{3}$ & 40 \\
\hline$m_{4}$ & 40 \\
\hline hidden state size at $\mathrm{C}_{1}^{1}$ & 5 \\
\hline hidden state size at $\mathrm{C}_{1}^{2}$ & 10 \\
\hline hidden state size at $\mathrm{C}_{1}^{3}$ & 10 \\
\hline hidden state size at $\mathrm{C}_{2}^{1}$ & 32 \\
\hline
\end{tabular}




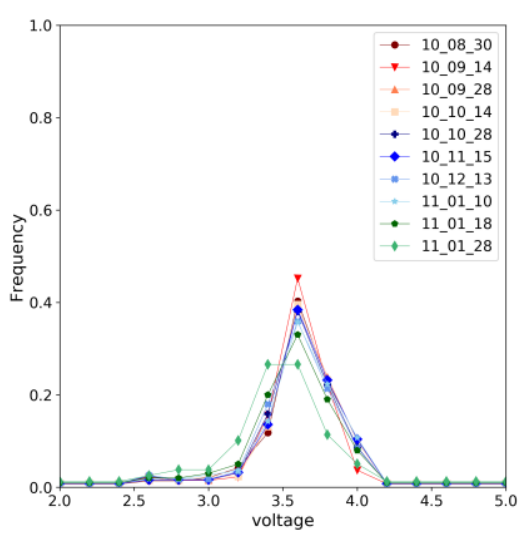

(a)

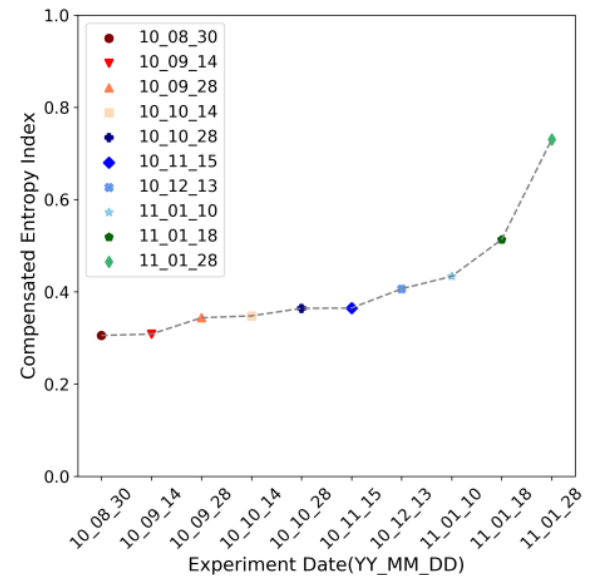

(b)

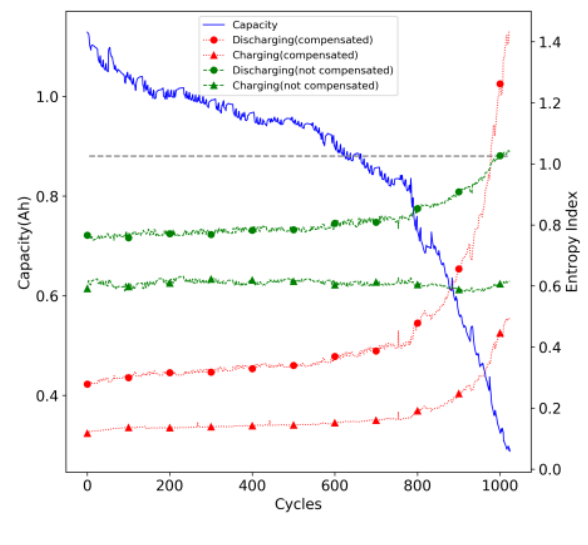

(c)

Figure 5. (a): Change of discharging voltage distribution of CS2_38. As the battery gets older, the distribution becomes wider. (b): The discharging time compensated entropy indices from (a). (c): The capacity degeneration curves and the entropy curves at discharging (circular mark) and charging (triangular mark) whether compensated or not.

Table 2. The results of error variation according to the sequence lengths and prediction cycles.

\begin{tabular}{|c|c|c|c|}
\hline $\begin{array}{c}\text { Sequence } \\
\text { Length }\end{array}$ & $\begin{array}{c}\text { Prediction } \\
\text { Cycle }\end{array}$ & $E_{[0: 40]}$ & $E_{\text {all }}$ \\
\hline 80 & 80 & 0.001197 & 0.000879 \\
\hline 100 & 80 & 0.001750 & 0.001315 \\
\hline 120 & 80 & 0.004682 & 0.003705 \\
\hline
\end{tabular}

$E_{a l l}$ is the mean of (prediction - target) $)^{2}$ of all cycles in prediction coverage while $E_{[0: 40]}$ is error of 40 cycles. Both errors are lowest if the sequence length is 80 . Figure 4 describes results including error per each prediction points and variance per 10 cycles. In all conditions, the error increases as the point far from the starting point is predicted; see the figure 4 (a), (b) and (c). The distribution of errors also gets bigger and bigger; see the figure 4 (d), (e) and (f). In figure 4 (a) and (b), the error increase rapidly after the prediction point 40 . It means prediction results in a column is more accurate until 40 cycles. Therefore, we determine the sequence length and prediction cycle in P-LSTM for RUL as 80 and 40, respectively.

\subsection{Results}

\subsubsection{Time Compensated Entropy Index}

Figure 5 shows the time compensated entropy index in CS2_38 according to the battery operating cycles. To simplify, one cycle per each experiment that were performed on different days is considered. Time compensated entropy indices of battery are calculated based on voltage distribution in Eq. (4). Figure 5 (a) describes discharging voltage distributions based on histogram which has 16 bins in different experiment dates. The distribution has a uniform distribution as the experiment continues. The wider distribution means, the more uncertain in expected measurement voltage. The uncertainty makes information entropy increase. In figure 5 (b), discharging time compensated entropy index (DE) from distributions in figure 5 (a) change as time goes by. Eq. (4) is used to calculate DE with $\alpha=50$. DE increases over time as the variance of voltage distribution increases. The change in time compensated entropy index until the maximum charge capacity reaches about 0.2 is shown in figure 5 (c). In figure 5 (c) we consider the variation in entropy indices depending on whether compensated or not about every cycle in CS2_38. When entropy index is obtained from Eq. (4) with a time compensated term, the tendency to increase with aging becomes more pronounced than without compensated term like in Eq. (3). As chargeable capacity decreases, time compensated entropy index in charging and discharging have drastic growth.

\subsubsection{Estimation SOH and RUL based on P-LSTM}

Through the designed P-LSTM for SOH and RUL, experiment to estimate the $\mathrm{SOH}$ per cycle and predict the future $\mathrm{SOH}$ sequence is conducted. The results of estimation of $\mathrm{SOH}$ in $\mathrm{C}_{1}^{1}$ from battery discharging information including entropy are described in the figure 6. CS2_36 dataset is used as test dataset. Predicting SOH based on battery measurement data can be done by SOH estimating column network and time compensated entropy indices. An additional experiment was conducted to estimate $\mathrm{SOH}$ using $\mathrm{CS} 2 \_33$ discharged with $0.5 \mathrm{C}$ current. In this case, CS2_34, CS2_36, CS2_37, $\mathrm{CS} 2 \_38$ are training datasets and $\mathrm{C}_{2} \_35$ is used as a validation dataset. For predicting near future $\mathrm{SOH}$, estimated 
SOH values from $\mathrm{C}_{1}^{1}$ and $\mathrm{C}_{1}^{2}$ are utilized. The results from two experiments with P-LSTM for SOH are in the table 3.

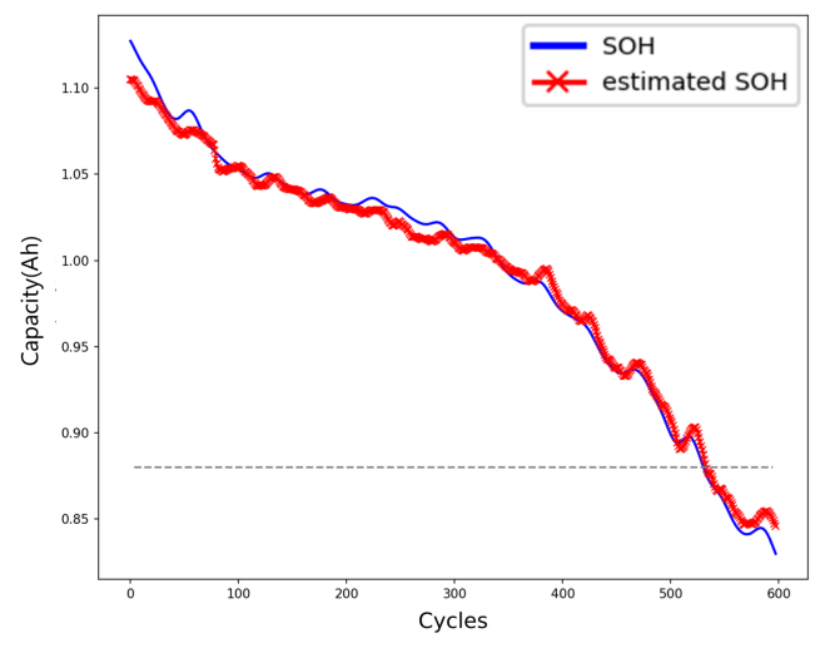

Figure 6. SOH estimation results with $\mathrm{CS} 2 \_36$.

Table 3. The results of $\mathrm{SOH}$ estimation from P-LSTM for SOH.

\begin{tabular}{|c|c|c|c|}
\hline $\begin{array}{l}\text { Test Case } \\
\text { (Discharge) }\end{array}$ & Column & $\begin{array}{c}\text { Prediction } \\
\text { Target }\end{array}$ & RMSE \\
\hline \multirow{3}{*}{$\begin{array}{c}\mathrm{CS} 236 \\
(1 \mathrm{C})\end{array}$} & $\mathrm{C}_{1}^{1}$ & $\mathrm{SOH}_{\mathrm{t}}$ & 0.0067 \\
\hline & $C_{1}^{2}$ & $\mathrm{SOH}_{\mathrm{t}: 20}$ & 0.0077 \\
\hline & $C_{1}^{3}$ & $\mathrm{SOH}_{\mathrm{t}: 40}$ & 0.0098 \\
\hline \multirow{3}{*}{$\begin{array}{c}\mathrm{CS} 2 \_33 \\
(0.5 \mathrm{C})\end{array}$} & $\mathrm{C}_{1}^{1}$ & $\mathrm{SOH}_{\mathrm{t}}$ & 0.0211 \\
\hline & $C_{1}^{2}$ & $\mathrm{SOH}_{\mathrm{t}: 20}$ & 0.0147 \\
\hline & $\mathrm{C}_{1}^{3}$ & $\mathrm{SOH}_{\mathrm{t}: 40}$ & 0.0209 \\
\hline \multirow{3}{*}{$\begin{array}{c}{ }^{*} \mathrm{CS} 2 \_33 \\
(0.5 \mathrm{C})\end{array}$} & $\mathrm{C}_{1}^{1}$ & $\mathrm{SOH}_{\mathrm{t}}$ & 0.0201 \\
\hline & $\mathrm{C}_{1}^{2}$ & $\mathrm{SOH}_{\mathrm{t}: 20}$ & 0.0267 \\
\hline & $\mathrm{C}_{1}^{3}$ & $\mathrm{SOH}_{\mathrm{t}: 40}$ & 0.0295 \\
\hline
\end{tabular}

Due to imbalance in datasets which have 4 datasets with $1 \mathrm{C}$ and 2 datasets with $0.5 \mathrm{C}$, RMSE results vary depending on the discharge current value. The result shows, for $1 \mathrm{C}$ discharging current datasets which are relatively abundant, the accuracy is relatively high while bad performance where $0.5 \mathrm{C}$ charging current dataset is used as the test dataset. The additional experiment, ${ }^{*} \mathrm{CS} 2 \_33$ on a table, is predicting $\mathrm{SOH}$ of battery discharged by $0.5 \mathrm{C}$ with the network trained by datasets only with discharging current of $1 \mathrm{C}$. Our method can be applied to several discharge currents through the current compensated term in Eq. (5). The result of the experiment under limited conditions shows that the proposed time compensated entropy index can be applied in an environment where the discharge current varies at each cycle. A more accurate estimation method and its application in a changing current environment during one operating cycle will be performed in future studies.

SOH prediction until EOL is generated from $\mathrm{C}_{2}^{1}$. Obtained near future $\mathrm{SOH}$ from $\mathrm{C}_{1}^{3}$ is followed by predicted $\mathrm{SOH}$ sequences. After a single prediction with $m_{4}$ length is performed, the prediction is utilized as an input to make repeated prediction. Two predictions are conducted in two cases. When CS2_36 is used as a test dataset, actual target $\mathrm{EOL}\left(\mathrm{EOL}_{l}\right)$ is at 504 th cycle and predicted EOL $\left(\mathrm{EOL}_{p}\right)$ is at 544th cycle. In the experiments with $\mathrm{CS}_{2} \_33, \mathrm{EOL}_{l}$ is at 544 and $\mathrm{EOL}_{p}$ is at 530th cycle. The difference between $\mathrm{EOL}_{l}$ and $\mathrm{EOL}_{p}$ at each case is 9 and 14, respectively. In the proposed P-LSTM structure, based on the experience obtained from $\mathrm{C}_{1}^{1}$, the model predicts near future $\mathrm{SOH}$ from $\mathrm{C}_{1}^{3}$ through $\mathrm{C}_{1}^{2}$. The RUL prediction is performed without prior learning before the prediction point as well as using not the actual $\mathrm{SOH}$ but estimated SOH. In Table 4, we can compare our results with from (Liu, Zhao, Peng, \& Hu, 2017) which conduct experiments with same battery dataset used in this paper. Proposed P-LSTM model uses estimated SOH to predict RUL while Liu et al. (2017) assumes label SOH is given as input data. Nevertheless, our model can predict EOL quite well.

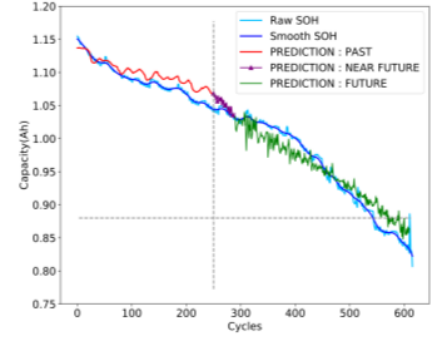

(a)

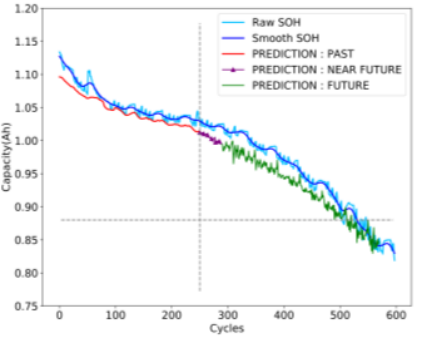

(b)
Figure 7. The results from P-LSTM for SOH and RUL. (a) and (b) are the results according to test datasets $0.5 \mathrm{C}$ and $1 \mathrm{C}$, respectively.

Table 4. RUL prediction results.

\begin{tabular}{|c|c|c|c|}
\hline Methods & $\begin{array}{c}\text { Test Case } \\
\text { (Discharge) }\end{array}$ & $\begin{array}{c}\text { Start } \\
\text { from }\end{array}$ & $\begin{array}{c}\text { Error } \\
\text { (cycle) }\end{array}$ \\
\hline Proposed P-LSTM & $\begin{array}{c}\text { CS2_33 } \\
(0.5 \mathrm{C})\end{array}$ & 250 & 14 \\
\cline { 2 - 4 } & $\begin{array}{c}\text { CS2_36 } \\
(1 \mathrm{C})\end{array}$ & 250 & 9 \\
\hline $\begin{array}{c}\text { LSTM } \\
\text { from Liu et al. (2017) }\end{array}$ & CS2_36 & 322 & 1 \\
\cline { 2 - 4 } & CS2_36 & 422 & 21 \\
\hline
\end{tabular}

Prediction on short-term $\mathrm{SOH}$ is critical factor in long-term RUL forecast. The prediction from the start point of the prediction to the EOL is performed independently without the help of the other columns, hence it is inevitably affected by 
the result of $\mathrm{C}_{1}^{3}$. In order to assure the influence of short-term $\mathrm{SOH}$ prediction results on RUL prediction, two prediction was conducted by using different $C_{1}^{2}$ inputs in the same network. The input vector of $\mathrm{C}_{1}^{2}$ includes 1) the output from $\mathrm{C}_{1}^{1}\left(\mathrm{C}_{1}^{1}\right.$ only) and 2$)$ the outputs from $\mathrm{C}_{1}^{1}$ and $\mathrm{C}_{1}^{3}\left(\mathrm{C}_{1}^{3}\right.$ with $\left.\mathrm{C}_{1}^{1}\right)$. Figure 8 shows the results of two predictions. Figure 8 and Table 5 show predicted near-future SOH from P-LSTM for $\mathrm{SOH}$ helps RUL prediction from P-LSTM for RUL get accuracy.

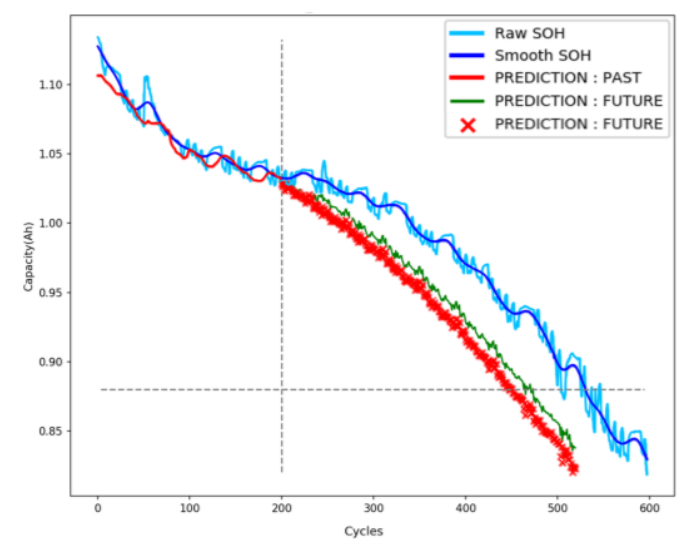

Figure 8. Prediction graph from 200th cycle of P-LSTM for RUL according to whether short-term future prediction results are included in input. red-cross mark: prediction with output of $\mathrm{C}_{1}^{1}$ only. green line: prediction with outputs from $C_{1}^{3}$ with $C_{1}^{1}$.

Table 5. RUL prediction error according to composition of the input vector of $\mathrm{C}_{2}^{1}$.

\begin{tabular}{|c|c|c|c|}
\hline case & $\begin{array}{c}\mathrm{EOL}_{p} \\
\text { (prediction) }\end{array}$ & $\begin{array}{c}\mathrm{EOL}_{l} \\
\text { (label) }\end{array}$ & $\begin{array}{c}\text { Error } \\
\text { (cycle) }\end{array}$ \\
\hline $\mathrm{C}_{1}^{3}$ with $\mathrm{C}_{1}^{1}$ & 485 & 504 & 19 \\
\hline $\mathrm{C}_{1}^{1}$ only & 449 & 504 & 55 \\
\hline
\end{tabular}

\section{Conclusion}

Estimating the aging information of lithium-ion batteries is an important research topic because degeneration of lithium ion batteries degrades system performance and lower reliability. However, lithium-ion batteries have difficulty in estimating the aging state due to the operation environment and internal complexity.

In this paper, we propose a time compensated entropy index that represents degree of aging by the concept of entropy. The time compensated entropy index confirm that the value increased according to degree of aging and can be used as an indicator to show the degradation phenomenon. We also proposed a P-LSTM structure that can predict battery $\mathrm{SOH}$ and RUL together using them. The proposed structure has the advantage of being able to perform two tasks in a single structure, away from the traditional SOH estimation and RUL prediction approaches. The accurate $\mathrm{SOH}$ estimation which is a preemptive condition of RUL prediction is performed with the time compensated entropy index and enable accurate $\mathrm{SOH}$ prediction of passing knowledge acquired to the $\mathrm{SOH}$ prediction column. The proposed model is able to predict SOH until EOL without any additional training with test data. We used the battery datasets from the CALCE battery to demonstrate that the structure can estimate and predicting $\mathrm{SOH}$.

The current research requires demonstration and improvement for experiments in complex discharge conditions. Therefore, the future issues are the application in more diverse environments and more investigation on optimization the number of $\mathrm{SOH}$ input and the RUL prediction sequences for the optimal parameter selections.

\section{ACKNOWLEDGEMENT}

This research was supported by the 'Project of e-Drive Train Platform Development for small and medium Commercial Electric Vehicles based on IoT Technology' of Korea Institute of Energy Technology Evaluation and Planning (KETEP) (20172010000420), sponsored by the Korea Ministry of Trade, Industry and Energy (MOTIE). This work was partly supported by Institute of Information \& communications Technology Planning \& Evaluation(IITP) grant funded by the Korea government(MSIT) (No.2019-000421, AI Graduate School Support Program). This work was supported, in part, by "Robocarechair: A Smart Transformable Robot for Multi-Functional Assistive Personal Care" Project, KEIT P0006886, of the Korea Evaluation Institute of Industrial Technology (KEIT). Battery testing is performed at Center for Advanced Life Cycle Engineering at the University of Maryland.

\section{REFERENCES}

Arora, P., White, R. E., \& Doyle, M. (1998). Capacity fade mechanisms and side reactions in lithium-ion batteries. Journal of the Electrochemical Society, 145(10), 3647-3667.

Vetter, J., Novák, P., Wagner, M. R., Veit, C., Möller, K. C., Besenhard, J. O., ... \& Hammouche, A. (2005). Ageing mechanisms in lithium-ion batteries. Journal of power sources, 147(1-2), 269-281.

Murnane, M., \& Ghazel, A. (2017). A closer look at state of charge (SOC) and state of health $(\mathrm{SOH})$ estimation techniques for batteries. http://www. analog. co $\mathrm{m} / \mathrm{media} / \mathrm{en} /$ technical-documentation/technicalarticles/A-Closer-Look-at-State-Of-Charge-and-StateHealth-Estimation-Techniques-.... pdf.

Ng, K. S., Moo, C. S., Chen, Y. P., \& Hsieh, Y. C. (2009). Enhanced coulomb counting method for estimating state-of-charge and state-of-health of lithium-ion batteries. Applied energy, 86(9), 1506-1511. 
Weng, C., Sun, J., \& Peng, H. (2014). A unified open-circuitvoltage model of lithium-ion batteries for state-of-charge estimation and state-of-health monitoring. Journal of power Sources, 258, 228-237.

D, Liu., X, Yin., Y, Song., W, Liu., and Y, Peng. (2017). An On-Line State of Health Estimation of Lithium-Ion Battery Using Unscented Particle Filter. IEEE Access, vol. 6. pp. 40990-41001. doi: 10.1109/ACCESS.2018.2854224

Peng, X., Zhang, C., Yu, Y., \& Zhou, Y. (2016). Battery remaining useful life prediction algorithm based on support vector regression and unscented particle filter. In 2016 IEEE International Conference on Prognostics and Health Management (ICPHM) (pp. 1-6). IEEE.

You, G. W., Park, S., \& Oh, D. (2017). Diagnosis of electric vehicle batteries using recurrent neural networks. IEEE Transactions on Industrial Electronics, 64(6), 48854893.

Zhang, Y., Xiong, R., He, H., \& Pecht, M. G. (2018). Long short-term memory recurrent neural network for remaining useful life prediction of lithium-ion batteries. IEEE Transactions on Vehicular Technology, 67(7), 5695-5705.

Rusu, A. A., Rabinowitz, N. C., Desjardins, G., Soyer, H., Kirkpatrick, J., Kavukcuoglu, K., ... \& Hadsell, R. (2016). Progressive neural networks. arXiv preprint arXiv:1606.04671.

Gideon, J., Khorram, S., Aldeneh, Z., Dimitriadis, D., \& Provost, E. M. (2017). Progressive neural networks for transfer learning in emotion recognition. arXiv preprint arXiv: 1706.03256.

Williard, N., He, W., Osterman, M., \& Pecht, M. (2013). Comparative analysis of features for determining state of health in lithium-ion batteries. Int. J. Progn. Health Manag, 4, 1-7.

Lorenzo, E., \& Labed, S. (2005). The battery voltage distribution: a possible tool for surveying the state of health of stand-alone PV systems. Progress in Photovoltaics: Research and Applications, 13(3), 251260.

Shannon, C. E. (1948). A mathematical theory of communication. Bell system technical journal,27(3), 379-423.

He, W., Williard, N., Osterman, M., \& Pecht, M. (2011). Prognostics of lithium-ion batteries based on DempsterShafer theory and the Bayesian Monte Carlo method. Journal of Power Sources, 196(23), 1031410321.

Kingma, D. P., \& Ba, J. (2014). Adam: A method for stochastic optimization. arXiv preprint arXiv:1412.6980.

Liu, Y., Zhao, G., Peng, X., \& Hu, C. (2017). Lithium-ion Battery Remaining Useful Life Prediction with Long Short-term Memory Recurrent Neural Network. Proceedings of the Annual Conference of the PHM Society, 8(7).

\section{BIOGRAPHIES}

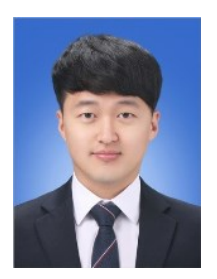

Taejun Bak received his B.S degrees in Electrical Engineering from Sungkyunkwan University in 2017. From 2016 to 2017, he was the engineer at ASML Korea. His research interests are in the areas of PHM based on intelligent systems, and time series analysis with machine learning. $\mathrm{He}$ is currently a Master's student at Sungkyunkwan University.

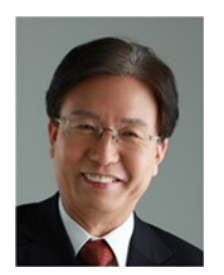

Sukhan Lee received the B.S. and M.S. degrees in electrical engineering from Seoul National University, South Korea, in 1972 and 1974, respectively, and the Ph.D. degree in electrical engineering from Purdue University, West Lafayette, IN, USA, in 1982. From 1983 to 1997 , he was with the Departments of Electrical Engineering and of Computer Science, University of Southern California. From 1990 to 1997, he was with the Jet Propulsion Laboratory, California Institute of Technology, as a Senior Member of the Technical Staff. From 1998 to 2003, he was the Executive Vice President, and also the Chief Research Officer with the Samsung Advanced Institute of Technology. He has been working as a Professor of information and communication engineering and WCU Professor of interaction science with Sungkyunkwan University, since 2003. He was designated the Dean of the Graduate School of Sungkyunkwan University, in 2011.He is also serving as the Director of the Intelligent Systems Research Institute. His research interests are in the areas of cognitive robotics, intelligent systems, and micro/nano electro-mechanical systems. Dr. Lee is currently a Fellow of the Korean National Academy of Science and Technology 